Conclusions Current calciphylaxis treatments alternatives aim to lower the serum calcium phosphate concentration thereby preventing, or even reversing, calcium phosphate oversaturation, precipitation and, finally, calcification. Administration of IV sodium thiosulfate, which sequesters calcium ions to form highly soluble calcium thiosulfate complexes, can prevent calcium phosphate precipitation.

No conflict of interest.

\section{CPC-146 TREATMENT OF GLIOBLASTOMA RECURRENCES: ROLE OF CHEMOTHERAPY - RETROSPECTIVE AND DESCRIPTIVE STUDY WITHIN 3 CENTRES OF THE N.E.N.O. GROUP (NORTHEAST NEURO-ONCOLOGY)}

doi:10.1136/ejhpharm-2013-000276.603

'P Bartecki-Lanher, ${ }^{2} \mathrm{~K}$ Hassani, ${ }^{3 P}$ Peruzzi, ${ }^{4} \mathrm{G}$ Noel, ${ }^{5} \mathrm{~L}$ Taillandier. ${ }^{1}$ Hôpital Bel Air CHR Metz-Thionville, Pharmacy, Thionville cedex, France; ${ }^{2}$ Hôpital Central, Pharmacy, Nancy, France; ${ }^{3}$ Hôpital Maison Blanche, Neurosurgery, Reims, France; ${ }^{4}$ Centre Paul Strauss, Radiotherapy, Strasbourg, France; ${ }^{5}$ Hôpital Central, Neurooncology, Nancy, France

Background Glioblastoma multiforme (GBM) are primary brain tumours that are currently incurable. Despite a well codified firstline treatment with concomitant radio-chemotherapy (temozolomide), recurrences of GBM occur and have limited treatment options. Furthermore, there is a lack of effective therapies and no standard relapse treatment. Anti-angiogenic drugs, such as bevacizumab, show encouraging results for patients with recurrences of high-grade gliomas.

Purpose To describe treatments of GBM relapses within three cities in northeast France: Nancy, Reims and Strasbourg. We especially tried to assess the impact of bevacizumab on survival endpoints.

Materials and Methods This is a retrospective study with GBM patients diagnosed between 2006 and 2008. Medical data describing the population and therapeutic oncology support were collected in each site from individual patient charts. Overall Survival (OS) and Progression Free Survival (PFS) were estimated by the Kaplan-Meier method and compared by the log-rank test.

Results Between 2006 and 2008, 321 patients were diagnosed with GBM, of whom 133 patients were treated for at least one recurrence. There were 95 males and 38 females; median age at diagnosis was 58. Main relevant signs of the initial tumour were intracranial hypertension and epilepsy. Initial treatment consisted for $64 \%$ of patients in surgical excision, and $86 \%$ of patients received conventional radio-chemotherapy followed by adjuvant temozolomide.

More than $50 \%$ of recurrences were diagnosed on both clinical and radiological grounds. Discarding palliative care, almost all patients with GBM relapse received chemotherapy: 95\% at first recurrence $(n=126 / 133), 95 \%$ at second recurrence $(n=69 / 73)$ and $100 \%$ at third recurrence $(n=26 / 26)$. Bevacizumab was used (alone or in association) in a third to half of cases.

In our population, neglecting the type of relapse treatment, median OS was 17.8 months [5-50 months]. When patients received bevacizumab at some point in their care, median OS was 20.2 months [7-50 months]. This OS is significantly different from the median OS observed without bevacizumab which was 13.5 months [5-41 months]. PFS until the second recurrence with bevacizumab was 5.5 months compared to 3.1 months without bevacizumab. PFS until the third recurrence with bevacizumab was 5 months against 2 months without bevacizumab. However, these results do not show bevacizumab providing significant PFS improvement, especially in the long term.

Conclusions Bevacizumab seems to improve OS in patients with GBM recurrences. However only prospective randomised studies will define the appropriate strategy treatment in recurrent glioblastoma. This work is one of the several projects of the NENO group which aims to standardise practise and build rational standards.

No conflict of interest.

\section{CPC-147 TREATMENT OF HEPATIC METASTASES FROM MELANOMA WITH IRINOTECAN LOADED IN ELUTING BEADS}

doi:10.1136/ejhpharm-2013-000276.604

${ }^{1} \mathrm{M}$ Ferrit, ${ }^{2} \mathrm{JE}$ Martinez, ${ }^{3 \mathrm{P}}$ Pardo, II Vallejo, ${ }^{2} \mathrm{E}$ Puerta, ' $\mathrm{N}$ Martinez, ${ }^{1} \mathrm{~A}$ Madrid, ${ }^{2} \mathrm{JA}$ Morales, ${ }^{2 P}$ Acosta, 'MA Calleja. 'University Hospital Virgen de las Nieves,

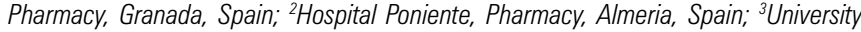
Hospital Virgen de las Nieves, Vascular Interventional Radiology, Granada, Spain

Background Chemoembolization of hepatic melanoma metastases refractory to treatment using irinotecan-loaded DC beads [embolic Drug-Eluting Beads]: a novel palliative treatment with which there is as yet little experience.

Purpose To show the progress of a clinical case of metastatic choroidal melanoma treated with irinotecan-loaded DC Beads.

Materials and Methods The pharmacy department loaded the particles with irinotecan ourselves and monitored the patient through the clinical history. The patient was a 38 -year-old man with stage IV choroidal melanoma in the left eye (2007).

Results In October 2011, 4 hepatic nodules were detected: 3 in segment VII $(23,25,11 \mathrm{~mm})$ and 1 in segment II $(16 \mathrm{~mm}) .2$ cycles of dacarbazine treatment $(1649 \mathrm{mg} \times 1$ day) stabilised the disease. The patient experienced emesis and diarrhoea. Given this intolerance and negative BRAFV600E mutation, ipilimumab reinforcement treatment was administered (225 mg $\times 1$ day q21days). After 4 cycles of ipilimumab, the disease stabilised for 5 months. In May 2012, an increase in size of the nodules was described and 6 new nodules in both hepatic lobes: segment II $(42 \times 34 \mathrm{~mm})$, IVb $(15 \mathrm{~mm})$, VII $(25,26$ and $61.4 \times 43 \mathrm{~mm})$ and VIII $(14 \mathrm{~mm})$ were observed. Surgery was rejected due to the presence of multinodular lesions and transarterial chemoembolization with irinotecan-loaded DC beads was attempted.

Hypervascular lesions were observed in the distal branches of the hepatic artery by bilobar hepatic arteriography using selective catheterization of both hepatic arteries. Subsequently, hepatic chemoembolization was performed by administering $100 \mathrm{mg}$ irinotecan-loaded beads (75-100 microns). After 2 cycles in each hepatic lobe, treatment response was assessed by the RECIST criteria. One month after the last chemoembolization, stable disease (no new nodules and arterial necrosis $<30 \%$ ) was confirmed. No immediate complications were observed, except for a slight elevation of hepatic enzymes that resolved.

Conclusions Hepatic chemoembolization using irinotecan-loaded beads is a viable alternative with good prognosis for hepatic metastases of choroidal melanoma. A higher concentration of chemotherapeutical drug is achieved within the hepatic lesions using lower doses of irinotecan, which therefore has less systemic impact.

No conflict of interest.

\section{CPC-148 TREATMENT OF SEVERE PSORIASIS WITH BIOLOGICALS}

doi:10.1136/ejhpharm-2013-000276.605

1 J Ruiz Gutiérrez, ${ }^{2 G}$ Roustán Gullón, 'P Calabuig Martínez, ${ }^{1} \mathrm{~A}$ Torralba Arranz. ${ }^{1}$ Hospital Universitario Puerta de Hierro Majadahonda, Pharmacy, Majadahonda (Madrid), Spain; ${ }^{2}$ Hospital Universitario Puerta de Hierro Majadahonda, Dermatology, Majadahonda (Madrid), Spain

Background Biological drugs are a relatively new class of treatment for severe psoriasis (SP).

Purpose To analyse the use and outcomes of biologicals in SP.

Materials and Methods Retrospective observational study for 23 months of patients with SP who had not previously received 